\title{
Discovery of a nitrogen-enhanced mildly metal-poor binary system: Possible evidence for pollution from an extinct AGB star
}

\author{
José G. Fernández-Trincado ${ }^{1,2,3}$, Ronald Mennickent ${ }^{3}$, Mauricio Cabezas ${ }^{4}$, Olga Zamora ${ }^{5,6}$, Sarah L. Martell ${ }^{7,8}$, \\ Timothy C. Beers ${ }^{9}$, Vinicius M. Placco ${ }^{9}$, David M. Nataf ${ }^{10}$, Szabolcs Mészáros ${ }^{11, \star}$, Dante Minniti ${ }^{12,13,14}$, \\ Dominik R. G. Schleicher ${ }^{3}$, Baitian Tang ${ }^{15}$, Angeles Pérez-Villegas ${ }^{16}$, Annie C. Robin ${ }^{2}$, and Céline Reylé ${ }^{2}$
}

(Affiliations can be found after the references)

Received 26 February 2019 / Accepted 2 September 2019

\begin{abstract}
We report the serendipitous discovery of a nitrogen-rich, mildly metal-poor $([\mathrm{Fe} / \mathrm{H}]=-1.08)$ giant star in a single-lined spectroscopic binary system found in the SDSS-IV Apache Point Observatory Galactic Evolution Experiment (APOGEE-2) survey, Data Release 14 (DR14). Previous work has assumed that two percent of halo giants with unusual elemental abundances have been evaporated from globular clusters, but other origins for their abundance signatures, including binary mass transfer, must also be explored. We present the results of an abundance reanalysis of the APOGEE-2 high-resolution near-infrared spectrum of 2M12451043+1217401 with the Brussels Automatic Stellar Parameter (BACCHUS) automated spectral analysis code. We manually re-derive the main element families, namely light elements $(\mathrm{C}, \mathrm{N})$, elements $(\mathrm{O}, \mathrm{Mg}, \mathrm{Si}), \mathrm{the}$ iron-peak element $(\mathrm{Fe}), s$-process element $(\mathrm{Ce})$, and light odd-Z element $(\mathrm{Al})$. Our analysis confirms the N-rich nature of 2M12451043+1217401, which has a $[\mathrm{N} / \mathrm{Fe}]$ ratio of +0.69 , and shows that the abundances of $\mathrm{C}$ and $\mathrm{Al}$ are slightly discrepant from those of a typical mildly metal-poor red giant branch star, but exhibit $\mathrm{Mg}, \mathrm{Si}, \mathrm{O}$ and $s$-process abundances $(\mathrm{Ce})$ of typical field stars. We also detect a particularly large variability in the radial velocity of this star over the period of the APOGEE- 2 observations; the most likely orbit fit to the radial velocity data has a period of $730.89 \pm 106.86$ days, a velocity semi-amplitude of $9.92 \pm 0.14 \mathrm{~km} \mathrm{~s}^{-1}$, and an eccentricity of $\sim 0.1276 \pm 0.1174$. These data support the hypothesis of a binary companion, which has probably been polluted by a now-extinct asymptotic giant branch star.
\end{abstract}

Key words. stars: abundances - stars: AGB and post-AGB - stars: evolution - stars: chemically peculiar - binaries: general techniques: spectroscopic

\section{Introduction}

It is clear that stellar populations with distinctive light-element abundance patterns (Bastian \& Lardo 2018; Fernández-Trincado et al. 2019a) are extremely common in globular clusters $(\mathrm{GCs})$, while metal-poor stars $([\mathrm{Fe} / \mathrm{H}] \lesssim-0.7)$ characterized by enhanced $\mathrm{N}([\mathrm{N} / \mathrm{Fe}] \gtrsim+0.5)$ and depleted $\mathrm{C}([\mathrm{C} / \mathrm{Fe}] \lesssim+0.15)$ are rarely found in the field (Johnson et al. 2007; Martell et al. 2011; Carollo et al. 2013). While currently still inconclusive, there is tantalizing evidence that stars with "anomalous chemistry" may be present beyond GC environments (e.g., Lind et al. 2015; Fernández-Trincado et al. 2016a, 2019b; Recio-Blanco et al. 2017).

To date, there have been a handful of stars fully characterized in terms of their chemistry and the chemical fingerprint of enriched second population ${ }^{1}$ stars (e.g., Martell et al. 2016; Fernández-Trincado et al. 2016b, 2017, 2019c,d; Schiavon et al. 2017), especially through observations of molecular ${ }^{16} \mathrm{OH}$, ${ }^{12} \mathrm{C}^{14} \mathrm{~N}$, and ${ }^{12} \mathrm{C}^{16} \mathrm{O}$ bands in the $H$ band of APOGEE (Majewski et al. 2017); these bands display the same chemical anomalies as stars in GCs and exhibit conspicuous anomalies of the CNO elements, most notably N.

These nitrogen-enhanced stars (hereafter N-rich stars) have received significant attention in recent years, primarily because they are believed to be likely relics of surviving galactic and/or

\footnotetext{
$\star$ Premium Postdoctoral Fellow of the Hungarian Academy of Sciences.

1 We refer to the second population as the groups of stars showing enhanced $\mathrm{Si}, \mathrm{N}$, and $\mathrm{Al}$, and depleted $\mathrm{C}$ and $\mathrm{O}$ abundances with respect to other field stars at the same metallicity $[\mathrm{Fe} / \mathrm{H}]$.
}

extragalactic (see Fernández-Trincado et al. 2017, for instance) GCs (Martell \& Grebel 2010), or now fully dissolved GCs (Fernández-Trincado et al. 2015a,b; Reis et al. 2018), and as such, play an important role in deciphering the early history of the Milky Way itself. Such stars are also characterized by significant star-to-star variation in the abundances of elements involved in proton-capture reactions, i.e., $\mathrm{C}, \mathrm{N}, \mathrm{O}, \mathrm{Mg}$, and $\mathrm{Al}$, among other.

Empirically, several subclasses exist within the population of N-rich stars, and these subclasses are defined by their stellar metallicity and their $\mathrm{Al}$ and/or $\mathrm{Mg}$ abundances. Most of the $\mathrm{N}$-rich stars in the bulge (e.g., Schiavon et al. 2017) exhibit intermediate aluminum $([\mathrm{Al} / \mathrm{Fe}] \lesssim+0.25)$ abundance ratios, similar to the thick disk. However, at higher metallicities, $[\mathrm{Fe} / \mathrm{H}]>$ -0.7 , Schiavon et al. (2017) identified a second group of N-rich stars chemically distinct from Milky Way stars across a variety of elements. Other groups of N-rich stars have been identified in the inner disk and the halo (Fernández-Trincado et al. 2016 b , 2017), with $[\mathrm{Al} / \mathrm{Fe}] \gtrsim+0.5$, which is significantly above the typical galactic level, across a range of metallicity. These stars are unlikely to have originated in tidally disrupted dwarf galaxies because of the rarity of Al-rich stars in current dwarf galaxies (Shetrone et al. 2003; Hasselquist et al. 2017). Fernández-Trincado et al. (2016b, 2017, 2019c) found that some $\mathrm{N}$-rich field stars are also $\mathrm{Mg}$-poor and Al-rich, similar to second-population stars in GCs, while some exhibit $\alpha$-element abundance levels to stars in the Milky Way at the same metallicity. There is also a subclass of N-rich stars with lower Al abundances of $[\mathrm{Al} / \mathrm{Fe}] \lesssim+0.1$, which tend to follow halo-like orbits with very little net rotation (Martell et al. 2016). 
The range of elemental abundances that can be derived from APOGEE $H$-band spectra makes it possible to identify and study these classes of stars in detail. With that information we can quantify their occurrence rates in the field and study their overall kinematic properties to better understand their origins.

The origins of most of these $\mathrm{N}$-rich stars are currently under investigation. Theories range from the formation of a $\mathrm{N}$-rich star via asymptotic giant branch (AGB) companion mass transfer or a massive evolved star, as suggested by Lennon et al. (2003), in which the former AGB companion has since become a faint white dwarf (see Pereira et al. 2017), to early accretion of GCs or dwarf spheroidal galaxies (dSPhs), as such stars appear chemically distinguishable from disk, halo, or bulge stars. However, the dynamical history of such stars remains unexplored to date. Measuring additional properties of the N-rich stars may help to further distinguish among them, provide clues to their origins, and/or identify more subpopulations, or more broadly help to understand GC formation and evolution.

In this study, we present the serendipitous discovery of a $\mathrm{N}$-rich star confirmed to be in a binary system with a compact object. The new object associated with 2 M12451043+1217401 is a nitrogen-enhanced and carbon-depleted metal-poor star with abundances of $\mathrm{Al}$ and $\mathrm{Mg}$ that are mildly discrepant from those of normal red giant branch (RGB) stars. We hypothesize that this star is likely to be an example of the result of mass transfer from a binary companion, which is now in the white dwarf stage of stellar evolution. The discovery of such stars helps guide models that attempt to explain the unusual elemental abundances over a wide range of metallicities.

This paper is outlined as follows. In Sect. 2, we present details and information regarding our serendipitous discovery in the APOGEE-2 data set. In Sect. 3, we describe and discuss the behavior of the measured light and heavy elements. In Sect. 4, we present our main conclusions.

\section{Data}

For our analysis we make use of the publicly available H-band spectra from the APOGEE-2 survey (Majewski et al. 2017). We draw on the latest data release, DR14 (Abolfathi et al. 2018), obtained with the multi-object high-resolution spectrograph APOGEE mounted at the Sloan $2.5 \mathrm{~m}$ Telescope (Gunn et al. 2006) at Apache Point Observatory, which began observation in 2014 as part of the Sloan Digital Sky Survey IV (Blanton et al. 2017). A detailed description of these observations, targeting strategy, data reduction, and APOGEE stellar-parameter estimates can be found in Zasowski et al. (2013, 2017), Nidever et al. (2015), Holtz man et al. (2015), Zamora et al. (2015), and García Pérez et al. (2016), respectively.

We start by searching for high-[N/Fe] outliers in the $[\mathrm{N} / \mathrm{Fe}]-$ $[\mathrm{Fe} / \mathrm{H}]$ abundance space in the first Payne data release of APOGEE abundances (hereafter Payne-APOGEE, see Ting et al. 2019). The Payne routine simultaneously derives best-fit values for all atmospheric parameters and abundances using neural networks; the parameter space of the training set is restricted to $[\mathrm{Fe} / \mathrm{H}] \gtrsim-1.5$. For each source, this routine only reports values for the measurement with the highest signal-to-noise ratio $(\mathrm{S} / \mathrm{N})$.

The sample was then restricted to the metallicity regions $-1.5 \lesssim[\mathrm{Fe} / \mathrm{H}] \lesssim-0.7$ and giant stars with $\log g<3.6$; this range encompasses the transition between the galactic thick disk and halo, following the same line of investigation as in Fernández-Trincado et al. (2017). Thus, we adopted the atmospheric parameters and $[\mathrm{C}, \mathrm{N}, \mathrm{O} / \mathrm{Fe}]$ and $[\mathrm{Fe} / \mathrm{H}]$ abundance ratios reported in the Payne catalog as the first-guess input parameters
Table 1. Comparison of the mean elemental abundances derived from our target 2M12451043+1217401 using the "abund" module in BACCHUS code, and those obtained with the Payne and ASPCAP pipeline.

\begin{tabular}{lccc}
\hline \hline Element & $\begin{array}{c}\text { BACCHUS }^{(\dagger)} \\
\text { Masseron et al. (2016) } \\
\sigma_{\text {total }}\end{array}$ & $\begin{array}{c}\text { Payne } \\
\text { Ting et al. (2019) }\end{array}$ & $\begin{array}{c}\text { ASPCAP } \\
\text { García Pérez et al. (2016) }\end{array}$ \\
\hline$T_{\text {eff }}$ & $4750.2 \pm 100 \mathrm{~K}$ & $4750.2 \mathrm{~K}$ & $4886.9 \pm 92.7 \mathrm{~K}$ \\
$\log g$ & $2.24 \pm 0.3$ & 2.24 & $2.22 \pm 0.08$ \\
$\xi_{t}$ & $1.86 \pm 0.05 \mathrm{~km} \mathrm{~s}^{-1}$ & $1.39 \mathrm{~km} \mathrm{~s}^{-1}$ & $1.44 \mathrm{~km} \mathrm{~s}^{-1}$ \\
{$[\mathrm{Fe} / \mathrm{H}]$} & $-1.08 \pm 0.14$ & -1.09 & $-1.12 \pm 0.01$ \\
{$[\mathrm{C} / \mathrm{Fe}]$} & $0.06 \pm 0.24$ & -0.23 & $-0.13 \pm 0.07$ \\
{$[\mathrm{~N} / \mathrm{Fe}]$} & $0.69 \pm 0.22$ & 0.66 & $0.74 \pm 0.09$ \\
{$[\mathrm{O} / \mathrm{Fe}]$} & $0.46 \pm 0.23$ & 0.22 & $0.22 \pm 0.07$ \\
{$[\mathrm{Al} / \mathrm{Fe}]$} & $0.04 \pm 0.16$ & -0.04 & $-0.05 \pm 0.08$ \\
{$[\mathrm{Mg} / \mathrm{Fe}]$} & $0.10 \pm 0.14$ & 0.22 & $0.22 \pm 0.04$ \\
{$[\mathrm{Si} / \mathrm{Fe}]$} & $0.22 \pm 0.12$ & 0.28 & $0.21 \pm 0.04$ \\
{$[\mathrm{Ce} / \mathrm{Fe}]$} & $0.49 \pm 0.20$ & $\ldots$ & $\ldots$ \\
\hline
\end{tabular}

Notes. The reported uncertainty for each chemical species in column 2 is: $\sigma_{\text {total }}=\sqrt{\sigma_{[\mathrm{X} / \mathrm{H}], T_{\mathrm{eff}}}^{2}+\sigma_{[\mathrm{X} / \mathrm{H}], \log g}^{2}+\sigma_{[\mathrm{X} / \mathrm{H}], \xi_{t}}^{2}+\sigma_{\text {mean }}^{2}}$. The Solar reference abundances are from Asplund et al. (2005) for light elements and Grevesse et al. (2015) for heavy elements. ${ }^{(\dagger)}$ The BACCHUS pipeline was used to derive the broadening parameters, metallicity, and chemical abundances.

into the Brussels Automatic Stellar Parameter (BACCHUS) code (Masseron et al. 2016) to derive the metallicity and chemical abundances reported in Table 1 . In addition to those literature values, we also systematically synthesized every element and line of 2M12451043+1217401 at high spectral resolution to compare with the Payne and ASPCAP determinations. We carried out a careful line selection and provided abundances based on a line-by-line differential approach in the atomic and molecular input data. The abundances that are derived for $2 \mathrm{M} 12451043+1217401$ using BACCHUS, found in Table 1, are generally in good agreement with those in the literature. In Appendix A, we describe the BACCHUS pipeline in more detail.

The high-[N/Fe] outlier sample itself was defined by fitting a fifth order polynomial to the run of $[\mathrm{N} / \mathrm{Fe}]$ with $[\mathrm{Fe} / \mathrm{H}]$, taking stars deviating from the fit by $+2.5 \sigma$. $2 \mathrm{M} 12451043+1217401$ is a high-[N/Fe] outlier in chemical space, as compared to the bulk of the disk, bulge, and halo stars in the Payne-APOGEE sample. The sample in Fig. 1 contains stars with $[\mathrm{C} / \mathrm{Fe}]<+0.15$ because higher $[\mathrm{C} / \mathrm{Fe}]$ abundance ratios are not typically found in $\mathrm{GCs}$, and we want to avoid contamination by $\mathrm{CH}$ stars, for instance.

In this first work, we only analyzed one star (2M12451043+ 1217401) that has large variability in radial velocity (see Appendix B for more details), out of 31 high-[N/Fe] outliers recently identified in Fernández-Trincado et al. (2019c), who analyzed the same data set. A comprehensive analysis of our new high- $[\mathrm{N} / \mathrm{Fe}]$ outliers is beyond the scope of this paper. But we searched in the literature for other high-[N/Fe] outliers in the more metal-poor $([\mathrm{Fe} / \mathrm{H}] \lesssim-0.7)$ population, identified in the APOGEE sample by Martell et al. (2016), Schiavon et al. (2017), and Fernández-Trincado et al. (2016b, 2017), with available chemical abundances in the Payne-APOGEE catalog, to verify that the Payne-APOGEE abundances and the polynomial fit used in this work are properly returning high-[N/Fe] outliers by recovering the most obvious known nitrogen-enhanced population. The results are shown in Fig. 1.

Figure 2 shows an example for a portion of the observed APOGEE spectrum of $2 \mathrm{M} 12451043+1217401$, where the ${ }^{12} \mathrm{C}^{14} \mathrm{~N}$ absorption feature is very strong. As a comparison, we also show the APOGEE spectrum of a field star with a normal nitrogen 

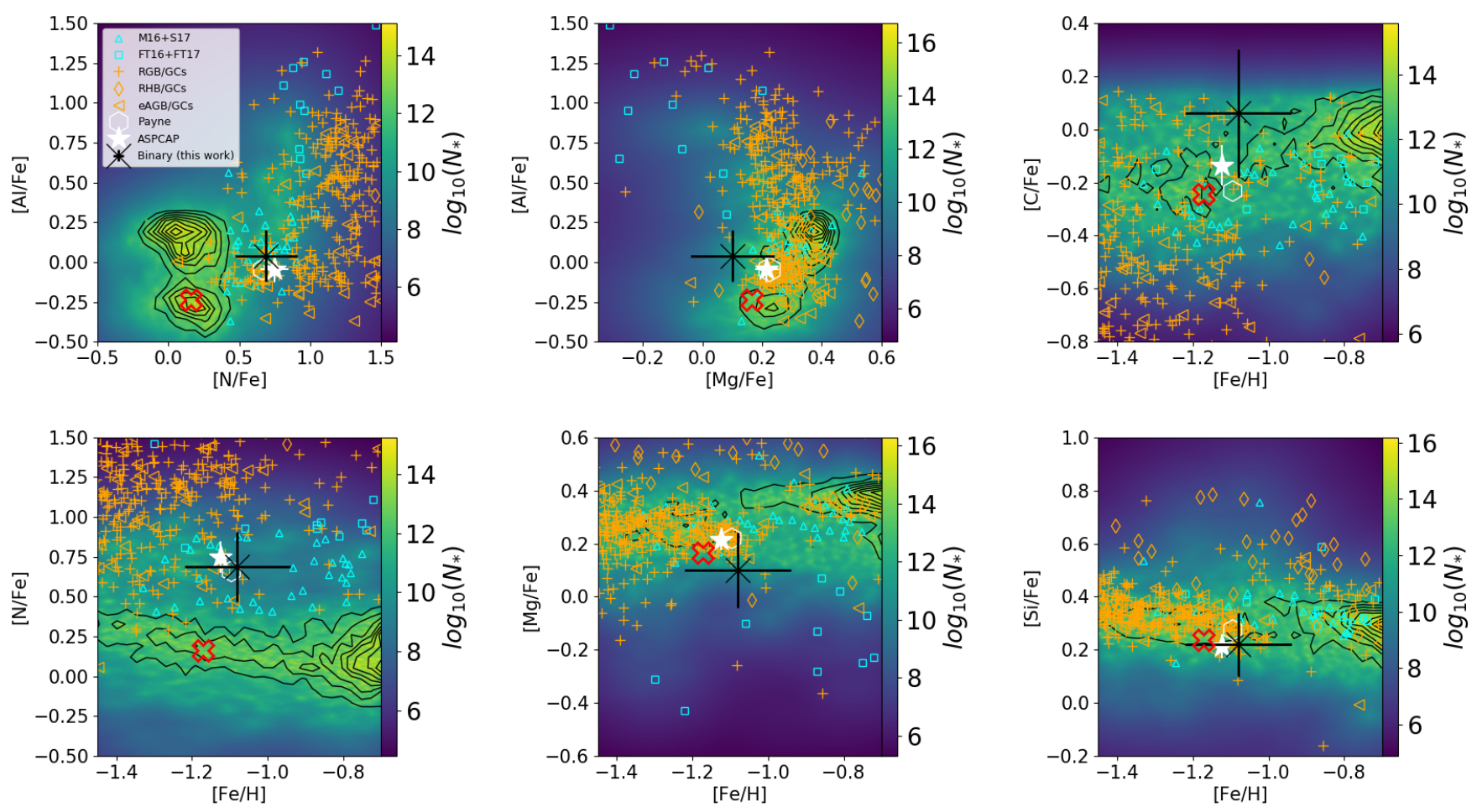

Fig. 1. Background color in each panel: Kernel density estimate smoothed distribution of $[\mathrm{Mg} / \mathrm{Fe}],[\mathrm{Al} / \mathrm{Fe}],[\mathrm{N} / \mathrm{Fe}],[\mathrm{C} / \mathrm{Fe}],[\mathrm{Si} / \mathrm{Fe}]$, and $[\mathrm{Fe} / \mathrm{H}]$ of Payne-APOGEE stars. The black contours show the density of Milky Way stars with normal N abundance $(\lesssim+0.5)$; the new N-rich star, 2M12451043+1217401 (see text), is highlighted with a black symbol. The plotted error bar shows the classical standard deviation derived from the different abundances of the different lines for each element, as reported in Table 1. The same field sample is compared to APOGEE/BACCHUS determinations for RGB, eAGB, and RHB stars (orange symbols) in GCs, M13 ([Fe/H] = -1.53 \pm 0.04$), \mathrm{M} 3([\mathrm{Fe} / \mathrm{H}]=-1.50 \pm 0.05), \mathrm{M} 5$ $([\mathrm{Fe} / \mathrm{H}]=-1.29 \pm 0.02), \mathrm{M} 107([\mathrm{Fe} / \mathrm{H}]=-1.02 \pm 0.02)$, and $\mathrm{M} 71([\mathrm{Fe} / \mathrm{H}]=-0.78 \pm 0.02)$. A sample of known N-rich stars is plotted using small cyan symbols: unfilled triangles for stars reported in M16-Martell et al. (2016) and S17-Schiavon et al. (2017) from Payne-APOGEE abundances, and unfilled squares for stars studied in FT16-Fernández-Trincado et al. (2016b) and FT17-Fernández-Trincado et al. (2017). The red "X" unfilled symbol indicates the typical abundance patterns of a normal RGB star, 2M12251747+1450078, with similar metallicity and stellar parameters as $2 \mathrm{M} 12451043+1217401$.

abundance $([\mathrm{N} / \mathrm{Fe}] \lesssim+0.5), 2 \mathrm{M} 15182930+0206378$, with stellar parameters and metallicity identical to that of the N-rich star. The N-rich star has remarkably stronger ${ }^{12} \mathrm{C}^{14} \mathrm{~N}$ lines, which can only mean that it has much higher nitrogen abundance. We conclude that the nitrogen abundance reported in this work, which is the basis for our identification of a new high- $[\mathrm{N} / \mathrm{Fe}]$ outlier in the Milky Way, is highly reliable, and detectable in 2M12451043+1217401

\section{Stellar parameters and chemical-abundance measurements}

The first estimates for stellar parameters for 2M12451043+ 1217401 , which we adopted in the present work, are $\left(T_{\text {eff }}\right.$, $\left.\log g, \xi_{t},[\mathrm{M} / \mathrm{H}]\right)=\left(4750.2 \mathrm{~K}, 2.24,1.86 \mathrm{~km} \mathrm{~s}^{-1},-1.09\right)$; we took these from Payne-APOGEE catalog. With this set of parameters, we performed an abundance analysis using the local thermodynamic equilibrium (LTE) abundance code BACCHUS (Masseron et al. 2016) and the plane-parallel, one-dimensional grid of MARCS model atmospheres (Gustafsson et al. 2008). The results are listed in Table 1 . The metallicity listed in column 2 of Table 1 is the average abundance of selected Fe I lines, and is in acceptable agreement with that determined from the ASPCAP and Payne-APOGEE pipelines.

In this work, we explore whether or not our chemicalabundance measurements have the ability to chemically tag stars associated with GCs by performing a chemical-tagging analysis.
Because we are interested in searching for high- $[\mathrm{N} / \mathrm{Fe}]$ outliers with chemical signatures typical of GC members, we focus on the abundances of $\mathrm{C}, \mathrm{N}, \mathrm{O}, \mathrm{Si}, \mathrm{Mg}$, and $\mathrm{Al}$. We also attempted to measure one additional element, namely Ce II, because it has three promising and detected lines (15784.8, 15958.4 and $16376.5 \AA$ ) in the $2 \mathrm{M} 12451043+1217401$ spectrum (see Fig. 3). This full set of abundance is the basis of our comparison with the literature values.

\subsection{Light-elements via spectrum synthesis: ${ }^{16} \mathrm{OH},{ }^{12} \mathrm{C}^{16} \mathrm{O}$, ${ }^{12} \mathrm{C}^{14} \mathrm{~N}, \mathrm{Mg} \mathrm{I}, \mathrm{Al} \mathrm{I}$, and Si l lines}

To put our results in context, we combined the chemicalabundance patterns $([\mathrm{Fe} / \mathrm{H}],[\mathrm{C} / \mathrm{Fe}],[\mathrm{N} / \mathrm{Fe}],[\mathrm{Mg} / \mathrm{Fe}],[\mathrm{Si} / \mathrm{Fe}]$, and $[\mathrm{Al} / \mathrm{Fe}])$ of $2 \mathrm{M} 12451043+1217401$, along with the chemical-abundance pattern of GC stars, other unusual giant stars (e.g., Martell et al. 2016; Fernández-Trincado et al. 2016b, 2017; Schiavon et al. 2017) from the APOGEE survey, and the entire Milky Way sample (see Fig. 1).

It is immediately clear from Fig. 1 that the chemicalabundance pattern of 2M12451043+1217401 appears to be distinguishable from galactic populations. Similarly, the elevated $[\mathrm{N} / \mathrm{Fe}]$ ratio of this star and the chemical distribution in the $\alpha$-elements appear most similar to those seen in the chemistry of normal stars in GCs (often called first-generation stars), and this is comparable to a few nitrogen-enhanced metalpoor field stars at similar metallicity. On the other hand, the 


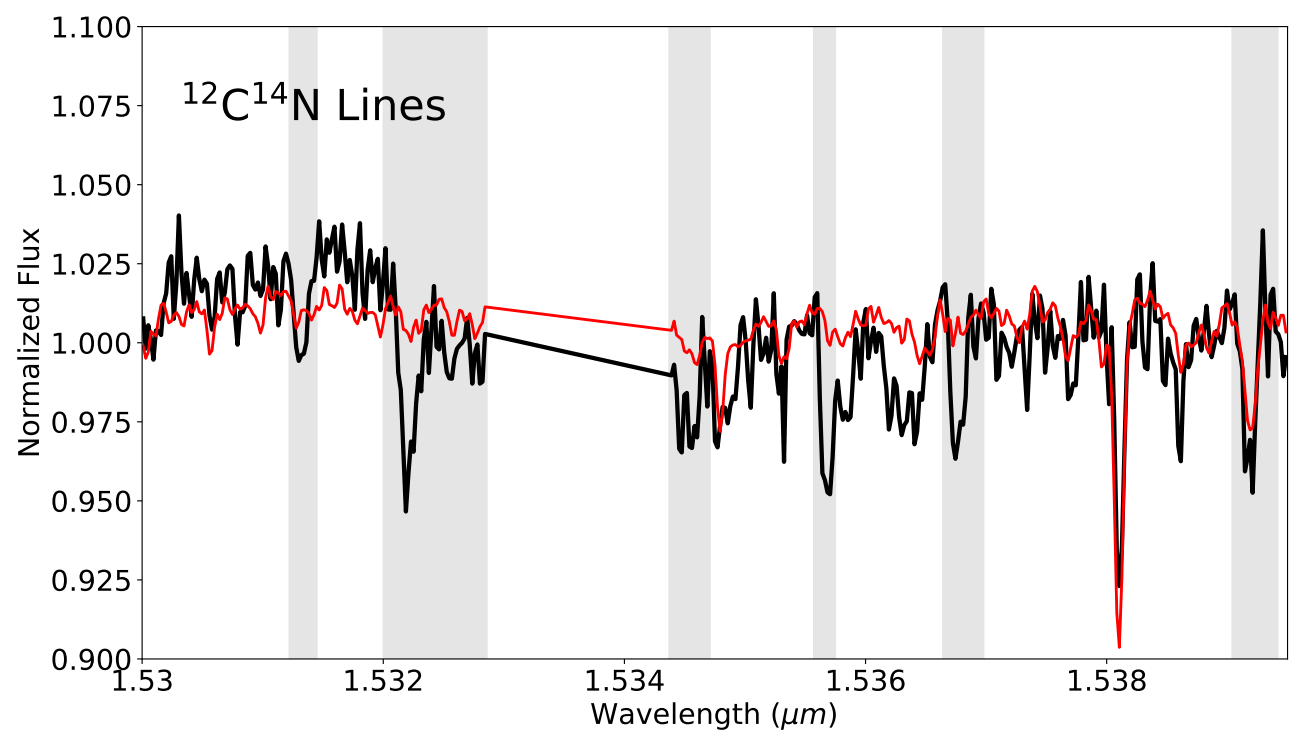

Fig. 2. Comparison between the spectrum of a normal (red line; APOGEE_ID: 2M12251747+1450078, $T_{\text {eff }}=4713.9 \mathrm{~K}, \log g=2.24$, $\xi_{t}=1.86 \mathrm{~km} \mathrm{~s}^{-1},[\mathrm{Fe} / \mathrm{H}]=-1.17 \mathrm{dex}$, and $S / N=637.8$ ) and our N-rich star (black line) with similar stellar parameters and similar metallicity. The gray vertical bands indicate the positions of ${ }^{12} \mathrm{C}^{14} \mathrm{~N}$ lines. moderately enhanced nitrogen level of this star, $[\mathrm{N} / \mathrm{Fe}] \sim+0.69$, suggests that this value is above the boundary that separates $\mathrm{N}$-rich stars $([\mathrm{N} / \mathrm{Fe}] \gtrsim+0.5)$ from objects with normal $\mathrm{N}$ abundances $([\mathrm{N} / \mathrm{Fe}] \lesssim+0.5)$, according to our strategy to separate these two population (see above text). This is strongly manifested in the $[\mathrm{Fe} / \mathrm{H}]$ versus $[\mathrm{N} / \mathrm{Fe}]$ plane in Fig. 1.

Figure 1 demonstrates that 2M12451043+1217401 seems to resemble the locus dominated by GC stars and the nitrogenenhanced metal-poor field stars discussed in Martell et al. (2016), Fernández-Trincado et al. (2016b), Schiavon et al. (2017), and Fernández-Trincado et al. (2017) in the [N/Fe]$[\mathrm{Fe} / \mathrm{H}]$ and $[\mathrm{Al} / \mathrm{Fe}]-[\mathrm{Fe} / \mathrm{H}]$ planes, which suggest that this star might have originated in GCs. However, another possible source for the abundance pattern in 2M12451043+1217401, given its radial velocity variability, is binary mass transfer from an possible AGB companion (see, e.g., Starkenburg et al. 2014). The mass range for the donor star is determined by the minimum mass for the third dredge-up, and by the onset of effective hot bottom burning, which burns $\mathrm{C}$ into $\mathrm{N}$ very effectively. It is important to note that without clear diagnostics such as mass, i.e., from an orbital solution, or a wide gamut of $s$ - $/ r$-process abundance patterns, the nature of the companion that polluted the N-rich binary star is not at all obvious from observations of $[\mathrm{X} / \mathrm{Fe}]$ or $[\mathrm{X} / \mathrm{H}]$ ratios (see below).

As can be seen in Fig. 1, this star occupies the same region of $\mathrm{Mg}-\mathrm{Al}$ abundance space as GC stars, without strong evidence of $\mathrm{Mg}-\mathrm{Al}$ cycles. The $\mathrm{Si}$ abundance is also moderately enriched, which is typical for GCs. While the abundance pattern of $2 \mathrm{M} 12451043+1217401$ seems consistent with GC stars in the $[\mathrm{N} / \mathrm{Fe}]-[\mathrm{Fe} / \mathrm{H}],[\mathrm{Mg} / \mathrm{Fe}]-[\mathrm{Fe} / \mathrm{H}],[\mathrm{Al} / \mathrm{Fe}]-[\mathrm{Mg} / \mathrm{Fe}]$, and $[\mathrm{Al} / \mathrm{Fe}]-[\mathrm{N} / \mathrm{FE}]$, this pattern is distinct from the overall APOGEE data set, which contains bulge, disk, and halo stars. We also see some distinction in carbon between 2 M12451043+1217401 and the main body of $\mathrm{N}$-normal stars and the GC population in that the N-normal stars and GCs stars typically have lower $[\mathrm{C} / \mathrm{Fe}]$ (for a given metallicity). In Fig. 1 we also compared the chemical-composition of a giant star in the main body of $\mathrm{N}$-normal stars with similar stellar parameters and a similar metallicity to $2 \mathrm{M} 12451043+1217401$; this clearly shows that our object has approximately the same $[\mathrm{Ce} / \mathrm{Fe}]$ (see below), $[\mathrm{Mg} / \mathrm{Fe}]$, $[\mathrm{Si} / \mathrm{Fe}]$, and $[\mathrm{Fe} / \mathrm{H}]$ abundance ratios as a normal giant star, but shows the most significant differences in their $[\mathrm{X} / \mathrm{Fe}]$ ratios, for $\alpha$-elements, N, C, and Al.

\subsection{Neutron-capture elements via spectrum synthesis: Ce II lines}

From a spectral synthesis of the Ce II lines in the APOGEE window, at $15784.8,15958.4$, and $16376.5 \AA$, we derive a cerium abundance ratio of $[\mathrm{Ce} / \mathrm{Fe}]=+0.49 \pm 0.20$, which has an uncertainty mainly driven by stellar parameters (see Table A.3), most sensitive to the surface gravity values. This represents a modest enhancement of the $s$-process in the $\mathrm{N}$-rich binary star, and is similar to the typical enhancement of $[\mathrm{Ce} / \mathrm{Fe}]$ found in field stars with similar metallicities.

Figure 3 shows three of the Ce II lines in 2M12451043+ 1217401 in the APOGEE window along with the synthetic fits to the spectrum. The Ce II lines are clearly detected and well fit. In the same figure the spectrum of $2 \mathrm{M} 12451043+1217401$ is compared to a field (normal) star with similar stellar parameters and with similar metallicity. This brief examination reassures us that the existence of a noticeably strengthened in the $s$-process in $2 \mathrm{M} 12451043+1217401$, in view of the similarity between to the two stars in all the other relevant parameters, can only mean that this star has mildly enhanced values of [Ce/Fe]. These values, however, are still comparable to the $s$-process content of a typical field star (see Fig. 4) and differ only notably on the basis of their nitrogen composition as illustrated in Figs. 2 and 4.

Figure 5 also shows that $2 \mathrm{M} 12451043+1217401$ exhibits $[\mathrm{Ce} / \mathrm{Fe}]$ ratios identical to typical RGB stars as seen in GC environments (e.g., Masseron et al. 2019; Nataf et al. 2019) at similar metallicity, with the peculiarity that $2 \mathrm{M} 12451043+1217401$ is clearly enhanced in nitrogen as compared to a $\mathrm{N}$-normal field star (see Fig. 2). We showed that 2M12451043+1217401 is a mildly metal-poor binary star characterized by an enhancement of nitrogen with a modest enhancement of the $s$-process elements. Therefore, we hypothesize that it is possible that the high $[\mathrm{N} / \mathrm{Fe}]$ abundance ratio simultaneous with the basic pattern in $\mathrm{Ce}, \mathrm{C}$, and $\mathrm{Al}$ could be due to pollution from a previous AGB companion which is now a white dwarf.

From a theoretical point of view, intermediate-mass AGB stars with masses of 3-8 $M_{\odot}$ may influence nucleosynthesis in which $\mathrm{N}$ is strongly enhanced by hot-bottom burning (HBB) at the expense of C (Masseron et al. 2010), and may be able to produce simultaneously a considerable amount of nitrogen (Cristallo et al. 2015). In this case, we are not measuring the original abundance of the red giant star, but these abundances reflect the chemical 

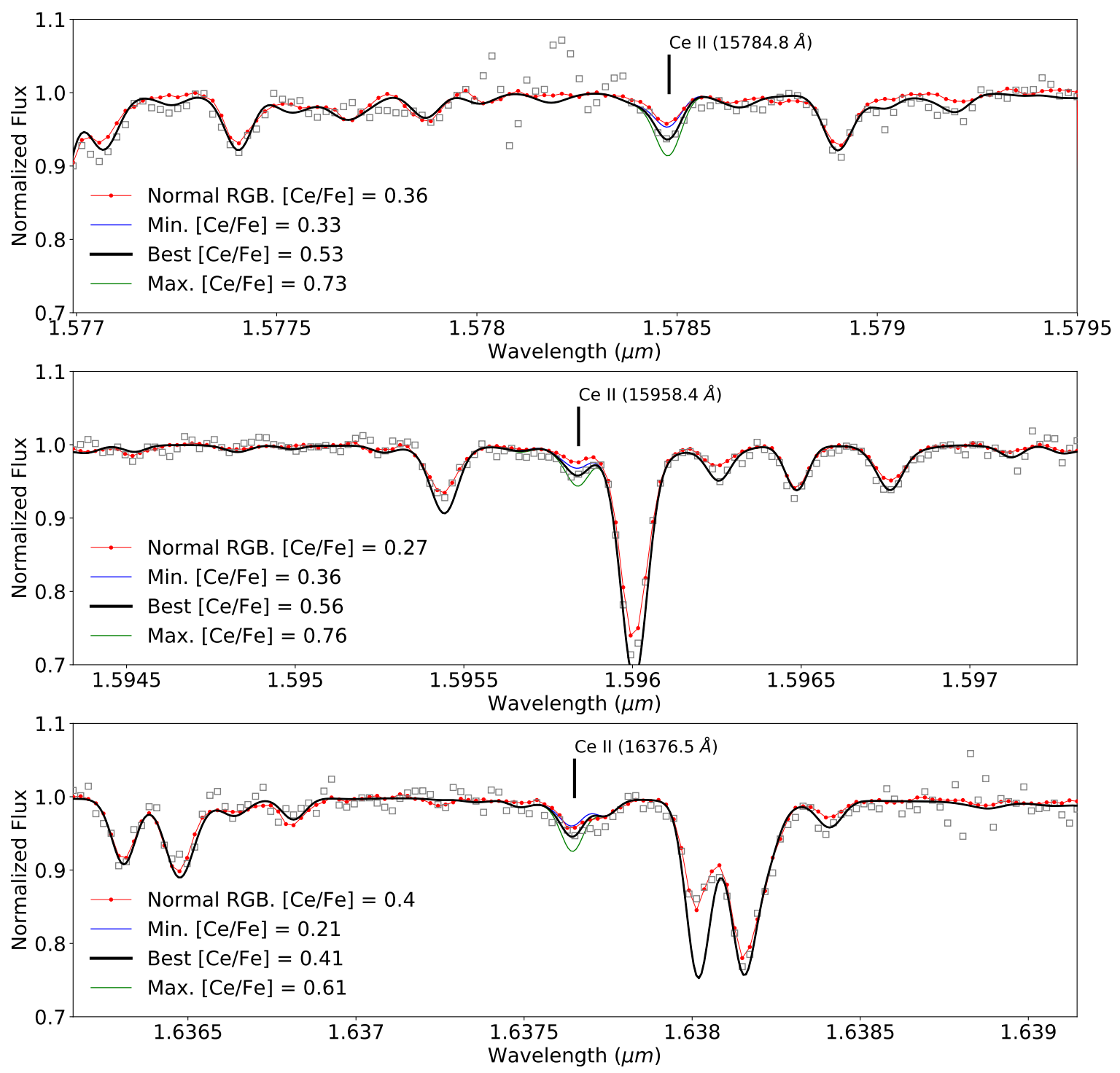

Fig. 3. Example spectra and the fitted synthesis of Ce II lines for 2M12451043+1217401 in the observed infrared spectrum (gray unfilled squares). The printed best-fitted abundance (black thick line) values might not be the same as in Table 1 because the table contains averaged values, not individual fits. The blue and green lines correspond to synthetic spectrum abundance choices that are offset from the best fit by \pm 0.2 dex. The spectrum of the N-rich star is compared to the spectrum of a normal star with [Ce/Fe] $=+0.34$ dex (the red line corresponds to APOGEE_ID: 2M12251747+1450078, labeled as Normal RGB), with similar stellar parameters.

composition of the companion, plus some degree of dilution with the convective envelope of the accreting star. To summarize, although the mechanism responsible for the $\mathrm{N}$ production could be attributed to pollution from an intermediate-mass AGB star, no current AGB models reproduce the trend observed in Fig. 4 for $2 \mathrm{M} 12451043+1217401$. Thus we cannot use $\mathrm{N}$ or Ce to place an additional constraint on the mass of the progenitor. Thus, a future inventory of the chemistry of this system, in particular, the elements formed by neutron-capture processes, would hint at the range of mass of the companion, and possibly help confirm or refute the association with an extinct AGB star.

We have shown that $2 \mathrm{M} 12451043+1217401$ exhibits radial velocity variation, which is consistent with being a binary. It is indeed possible that an intermediate-mass companion has undergone its AGB phase and dumped shell-nucleosynthesis processed material onto the observed star, in a similar fashion to what happens for $\mathrm{CH}$ and CEMP stars (e.g., Cristallo et al. 2016), thereby increasing the content of N, C, and Al, as compared to a typical field RGB star (see Fig. 4).

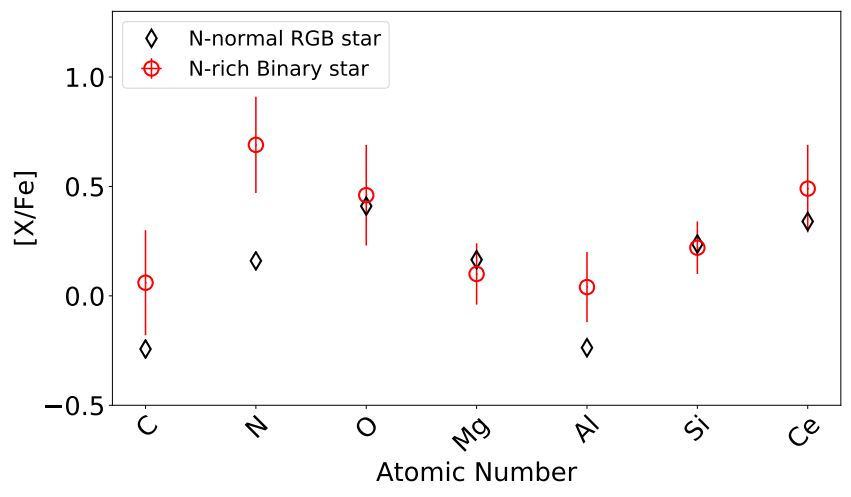

Fig. 4. Chemical abundance pattern of $2 \mathrm{M} 12451043+121740$ (N-rich Binary star), for elements $\mathrm{X}$, where $\mathrm{X}$ is delineated at the label of the figure. Each determined abundance is shown as an open red circle. These abundances are compared to an N-normal RGB star (black diamond symbols) with similar atmospheric parameters and similar metallicity as the N-rich Binary star. 


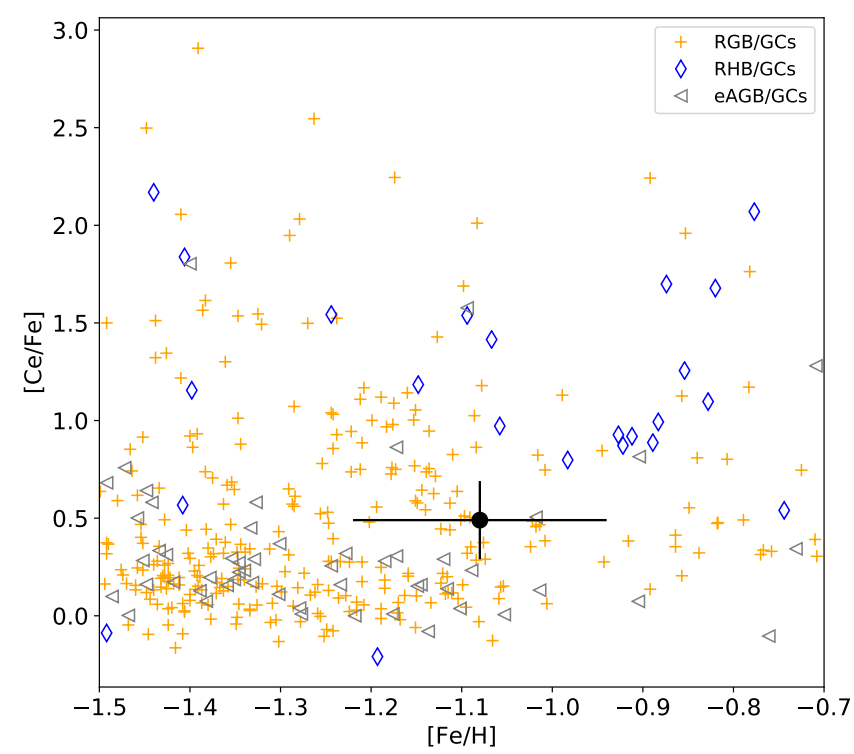

Fig. 5. Abundances of $[\mathrm{Fe} / \mathrm{H}]$ vs. $[\mathrm{Ce} / \mathrm{Fe}]$ in a large number of galactic GC stars (M107, M71, M5, M3 and M13) from Masseron et al. (2019). The N-rich binary star $(2 \mathrm{M} 12451043+1217401)$ with estimated Ce II indicated by a black filled circle.

\section{Concluding remarks}

In this work, we communicate the serendipitous discovery of an unusual red giant star, which shows significantly enhanced $[\mathrm{N} / \mathrm{Fe}]$ among metal-poor field stars. Based on high-resolution near-infrared spectroscopic data from the APOGEE-2 survey, we determined the atmospheric parameters, spectrostopic distance, radial velocity variability, abundances of light elements $(\mathrm{C}, \mathrm{N}$, $\mathrm{O}, \mathrm{Mg}, \mathrm{Al}$, and $\mathrm{Si}$ ), and the elements created by the $s$-process (Ce II) for 2M12451043+1217401.

Combining the large radial velocity variation and nitrogen overabundance, we hypothesise that an possible AGB-binary system may produce a $[\mathrm{N} / \mathrm{Fe}]$ overabundance in some $\mathrm{N}$-rich stars within the Milky Way. This is the case because mass transfer happened in the past and the star is a normal RGB star in which we see the effects due to the pollution of a companion that has undergone its AGB phase and dumped shell-nucleosynthesis processed material onto the observed star; i.e., the AGB companion deposits its N-rich outer layers onto its RGB companion through accreting winds, the binary system produces $\mathrm{N}$ (destroying $\mathrm{C}$ in the process), which is then mixed throughout the RGB envelope. The companion star in the system is now a possible white dwarf. A future inventory of the chemistry of this binary system, in particular the elements involved in the neutroncapture reactions (i.e., like hs/ls) such as those found in AGB stars, which are independent of the amount of pollution of the observed star undergoes, is crucial to understand the origin of this unique object and place constraints on the range of mass of the companion.

A dynamical study of $2 \mathrm{M} 12451043+1217401$ shows that this system has a retrograde motion with a highly eccentric orbit, which is consistent with the galactic inner halo population. The results are described in Appendix $\mathrm{C}$ and illustrated in Fig. C.1. By this we mean that this system was formed early on before the inner halo formation, or else the system formed together with the inner-halo. The present paucity of halo binary $\mathrm{N}$-enriched stars could therefore also have implications for the binary fraction in the field, which to date has been difficult to determine. This study further supports the idea that AGB stars could be the key players in the pollution of ${ }^{12} \mathrm{C}^{14} \mathrm{~N}$ via a slow stellar wind that has polluted the RGB, and thus their atmospheric chemistry reflects some of the yields from an AGB star.

Acknowledgements. We thank the referee for her/his work that improved the manuscript. J.G.F-T is supported by FONDECYT No. 3180210 and the European COST Action CA16117 (ChETEC) project No 41736. R.E.M. acknowledges project fondecyt 1190621, and also acknowledges support by VRIDEnlace 218.016.004-1.0 and the Chilean Centro de Excelencia en Astrofísica y Tecnologías Afines (CATA) BASAL grant AFB-170002. M.C. acknowledges support by Astronomical Institute of the Czech Academy of Sciences through the project RVO 67985815. O.Z. acknowledge support by the MINECO under grant AYA-2017-88254-P. SzM has been supported by the Premium Postdoctoral Research Program of the Hungarian Academy of Sciences, and by the Hungarian NKFI Grants K-119517 and GINOP-2.3.2-15-2016-00003 of the Hungarian National Research, Development and Innovation Office. D.M. acknowledges support from FONDECYT Regular grant No. 1170121, and the kind hospitality of the Osservatorio di Capodimonte/INAF, Italy. T.C.B. and V.M.P. acknowledge partial support for this work from grant PHY 14-30152; Physics Frontier Center/JINA Center for the Evolution of the Elements (JINA-CEE), awarded by the US National Science Foundation. APV acknowledges a FAPESP for the postdoctoral fellowship grant no. 2017/15893-1 and the DGAPA-PAPIIT grant IG100319. B.T. gratefully acknowledges support from National Natural Science Foundation of China under grant No. U1931102 and support from the hundredtalent project of Sun Yat-sen University. Funding for the GravPot16 software has been provided by the Centre national d'études spatiales (CNES) through grant 0101973 and UTINAM Institute of the Université de Franche-Comté, supported by the Région de Franche-Comté and Institut des Sciences de l'Univers (INSU). Simulations have been executed on computers from the Utinam Institute of the Université de Franche-Comté, supported by the Région de Franche-Comté and Institut des Sciences de l'Univers (INSU), and on the supercomputer facilities of the Mésocentre de calcul de Franche-Comté. Funding for the Sloan Digital Sky Survey IV has been provided by the Alfred P. Sloan Foundation, the U.S. Department of Energy Office of Science, and the Participating Institutions. SDSS-IV acknowledges support and resources from the Center for High-Performance Computing at the University of Utah. The SDSS web site is www.sdss.org. SDSS-IV is managed by the Astrophysical Research Consortium for the Participating Institutions of the SDSS Collaboration including the Brazilian Participation Group, the Carnegie Institution for Science, Carnegie Mellon University, the Chilean Participation Group, the French Participation Group, Harvard-Smithsonian Center for Astrophysics, Instituto de Astrofisica de Canarias, The Johns Hopkins University, Kavli Institute for the Physics and Mathematics of the Universe (IPMU)/University of Tokyo, Lawrence Berkeley National Laboratory, Leibniz Institut für Astrophysik Potsdam (AIP), MaxPlanck-Institut für Astronomie (MPIA Heidelberg), Max-Planck-Institut für Astrophysik (MPA Garching), Max-Planck-Institut für Extraterrestrische Physik (MPE), National Astronomical Observatory of China, New Mexico State University, New York University, University of Dame, Observatório Nacional/MCTI, The Ohio State University, Pennsylvania State University, Shanghai Astronomical Observatory, United Kingdom Participation Group, Universidad Nacional Autónoma de México, University of Arizona, University of Colorado Boulder, University of Oxford, University of Portsmouth, University of Utah, University of Virginia, University of Washington, University of Wisconsin, Vanderbilt University, and Yale University.

\section{References}

Abolfathi, B., Aguado, D. S., Aguilar, G., et al. 2018, ApJS, 235, 42 Alvarez, R., \& Plez, B. 1998, A\&A, 330, 1109

Asplund, M., Grevesse, N., \& Sauval, A. J. 2005, in Cosmic Abundances as Records of Stellar Evolution and Nucleosynthesis, eds. T. G. Barnes, \& F. N. Bash, ASP Conf. Ser., 336, 25

Bailer-Jones, C. A. L., Rybizki, J., Fouesneau, M., Mantelet, G., \& Andrae, R. 2018, AJ, 156, 58

Bastian, N., \& Lardo, C. 2018, ARA\&A, 56, 83

Blanton, M. R., Bershady, M. A., Abolfathi, B., et al. 2017, AJ, 154, 28

Brunthaler, A., Reid, M. J., Menten, K. M., et al. 2011, Astron. Nachr., 332, 461

Carollo, D., Martell, S. L., Beers, T. C., \& Freeman, K. C. 2013, ApJ, 769, 87

Charbonneau, P. 1995, ApJS, 101, 309

Cristallo, S., Straniero, O., Piersanti, L., \& Gobrecht, D. 2015, ApJS, 219, 40

Cristallo, S., Karinkuzhi, D., Goswami, A., Piersanti, L., \& Gobrecht, D. 2016, ApJ, 833, 181

Cunha, K., Smith, V. V., Hasselquist, S., et al. 2017, ApJ, 844, 145 
Fehlberg, E. 1968, NASA Technical Report, 315

Fernández-Trincado, J. G., Robin, A. C., Vieira, K., et al. 2015a, A\&A, 583, A76 Fernández-Trincado, J. G., Vivas, A. K., Mateu, C. E., et al. 2015b, A\&A, 574, A15

Fernández-Trincado, J. G., Robin, A. C., Reylé, C., et al. 2016a, MNRAS, 461, 1404

Fernández-Trincado, J. G., Robin, A. C., Moreno, E., et al. 2016b, ApJ, 833, 132

Fernández-Trincado, J. G., Zamora, O., García-Hernández, D. A., et al. 2017 ApJ, 846, L2

Fernández-Trincado, J. G., Zamora, O., Souto, D., et al. 2019a, A\&A, 627, A178

Fernández-Trincado, J. G., Moreno, E., \& Pérez-Villegas, A. 2019b, MNRAS submitted [arXiv:1904.05370]

Fernández-Trincado, J. G., Beers, T. C., Tang, B., et al. 2019c, MNRAS, 488, 2864

Fernández-Trincado, J. G., Beers, T. C., Placco, V. M., et al. 2019d, ApJL, submitted [arXiv: 1904.05884$]$

Gaia Collaboration (Brown, A. G. A., et al.) 2018, A\&A, 616, A1

García Pérez, A. E., Allende Prieto, C., Holtzman, J. A., et al. 2016, AJ, 151, 144

González Hernández, J. I., \& Bonifacio, P. 2009, A\&A, 497, 497

Grevesse, N., Scott, P., Asplund, M., \& Sauval, A. J. 2015, A\&A, 573, A27

Gunn, J. E., Siegmund, W. A., Mannery, E. J., et al. 2006, AJ, 131, 2332

Gustafsson, B., Edvardsson, B., Eriksson, K., et al. 2008, A\&A, 486, 951

Hasselquist, S., Shetrone, M., Smith, V., et al. 2017, ApJ, 845, 162

Hawkins, K., Masseron, T., Jofré, P., et al. 2016, A\&A, 594, A43

Holtzman, J. A., Shetrone, M., \& Johnson, J. A. 2015, AJ, 150, 148

Johnson, J. A., Herwig, F., Beers, T. C., \& Christlieb, N. 2007, ApJ, 658 1203

Lennon, D. J., Dufton, P. L., \& Crowley, C. 2003, A\&A, 398, 455

Lind, K., Koposov, S. E., Battistini, C., et al. 2015, A\&A, 575, L12

Majewski, S. R., Zasowski, G., \& Nidever, D. L. 2011, ApJ, 739, 25

Majewski, S. R., Schiavon, R. P., Frinchaboy, P. M., et al. 2017, AJ, 154, 94

Martell, S. L., \& Grebel, E. K. 2010, A\&A, 519, A14

Martell, S. L., Smolinski, J. P., Beers, T. C., \& Grebel, E. K. 2011, A\&A, 534, A136

Martell, S. L., Shetrone, M. D., Lucatello, S., et al. 2016, ApJ, 825, 146

Masseron, T., Johnson, J. A., Plez, B., et al. 2010, A\&A, 509, A93

Masseron, T., Merle, T., \& Hawkins, K. 2016, Astrophysics Source Code Library [record ascl:1605.004]

Masseron, T., García-Hernández, D. A., Mészáros, S., et al. 2019, A\&A, 622, A191

Mennickent, R. E., Kołaczkowski, Z., Djurasevic, G., et al. 2012, MNRAS, 427, 607

Mennickent, R. E., Kołaczkowski, Z., Soszyński, I., Cabezas, M., \& Garrido, H. E. 2018a, MNRAS, 473, 4055

Mennickent, R. E., Rivinius, T., Cidale, L., Soszyński, I., \& Fernández-Trincado, J. G. 2018b, PASP, 130, 094204

Nataf, D. M., Wyse, R. F. G., Schiavon, R. P., et al. 2019, AJ, 158, 14

Nidever, D. L., Holtzman, J. A., Allende Prieto, C., et al. 2015, AJ, 150, 173

Pereira, C. B., Smith, V. V., Drake, N. A., et al. 2017, MNRAS, 469, 774

Plez, B. 2012, Astrophysics Source Code Library [record ascl:1205.004]

Price-Whelan, A. M., Hogg, D. W., Rix, H.-W., et al. 2018, AJ, 156, 18

Queiroz, A. B. A., Anders, F., Santiago, B. X., et al. 2018, MNRAS, 476 2556

Recio-Blanco, A., Rojas-Arriagada, A., de Laverny, P., et al. 2017, A\&A, 602, L14
Reis, I., Poznanski, D., Baron, D., Zasowski, G., \& Shahaf, S. 2018, MNRAS, 476, 2117

Schiavon, R. P., Zamora, O., Carrera, R., et al. 2017, MNRAS, 465, 501

Shetrone, M., Venn, K. A., Tolstoy, E., et al. 2003, AJ, 125, 684

Smith, V. V., Cunha, K., Shetrone, M. D., et al. 2013, ApJ, 765, 16

Starkenburg, E., Shetrone, M. D., McConnachie, A. W., \& Venn, K. A. 2014, MNRAS, 441, 1217

Ting, Y.-S., Conroy, C., Rix, H.-W., \& Cargile, P. 2019, ApJ, 879, 69

Zamora, O., García-Hernández, D. A., Allende Prieto, C., et al. 2015, AJ, 149, 181

Zasowski, G., Johnson, J. A., Frinchaboy, P. M., et al. 2013, AJ, 146, 81

Zasowski, G., Cohen, R. E., Chojnowski, S. D., et al. 2017, AJ, 154, 198

1 Instituto de Astronomía y Ciencias Planetarias, Universidad de Atacama, Copayapu 485, Copiapó, Chile

2 Institut Utinam, CNRS UMR 6213, Université Bourgogne-FrancheComté, OSU THETA Franche-Comté, Observatoire de Besançon, BP 1615, 25010 Besançon Cedex, France e-mail: jfernandezt87@gmail.com, jfernandez@obs-besancon.fr

3 Departamento de Astronomía, Casilla 160-C, Universidad de Concepción, Concepción, Chile

4 Astronomical Institute of the Academy of Sciences of the Czech Republic, Boční II 1401/1, 14100 Prague, Czech Republic

5 Instituto de Astrofísica de Canarias, Vía Láctea s/n, 38205 La Laguna, Tenerife, Spain

6 Departamento de Astrofísica, Universidad de La Laguna (ULL), 38206 La Laguna, Tenerife, Spain

7 School of Physics, University of New South Wales, Sydney, NSW 2052, Australia

8 Centre of Excellence for Astrophysics in Three Dimensions (ASTRO-3D), Sydney, Australia

9 Department of Physics and JINA Center for the Evolution of the Elements, University of Notre Dame, Notre Dame, IN 46556, USA

10 Center for Astrophysical Sciences and Department of Physics and Astronomy, The Johns Hopkins University, Baltimore, MD 21218, USA

11 ELTE Eötvös Loránd University, Gothard Astrophysical Observatory, Szombathely, Hungary

12 Depto. de Cs. Físicas, Facultad de Ciencias Exactas, Universidad Andrés Bello, Av. Fernández Concha 700, Las Condes, Santiago, Chile

13 Millennium Institute of Astrophysics, Av. Vicuna Mackenna 4860, 782-0436 Santiago, Chile

14 Vatican Observatory, V00120 Vatican City State, Italy

15 School of Physics and Astronomy, Sun Yat-sen University, 519082 Zhuhai, PR China

16 Universidade de São Paulo, IAG, Rua do Matão 1226, Cidade Universitária, 05508-900 São Paulo, Brazil 


\section{Appendix A: Line-by-line abundances}

Tables A.1 and A.2 list the atomic and molecular lines used to derived abundances from a line-by-line differential analysis using the current version of the BACCHUS code (see Masseron et al. 2016), which relies on the radiative transfer code Turbospectrum (Alvarez \& Plez 1998; Plez 2012) and the MARCS model atmosphere grid (Gustafsson et al. 2008). For each element and each line, the abundance determination proceeds as in Hawkins et al. (2016), and is summarized in this appendix for guidance: (i) A spectrum synthesis, using the full set of (atomic and molecular) lines, is used to find the local continuum level via a linear fit; (ii) cosmic and telluric rejections are performed; (iii) the local $\mathrm{S} / \mathrm{N}$ is estimated; (iv) a series of flux points contributing to a given absorption line are automatically selected; and (v) abundances are then derived by comparing the observed spectrum with a set of convolved synthetic spectra characterized by different abundances. Four different abundance determinations are used: (i) line-profile fitting; (ii) a core line-intensity comparison; (iii) a global goodness-of-fit estimate; and (iv) equivalent width comparison. Each diagnostic yields validation flags. Based on these flags, a decision tree then rejects the line or accepts it, keeping the best-fit abundance. We adopted the $\chi^{2}$ diagnostic for the abundance decision because it is the most robust. However, we store the information from the other diagnostics, including the standard deviation between all four methods. The linelist used in this work is the latest internal DR14 atomic/molecular linelist (linelist.20170418), and the Ce II lines from Cunha et al. (2017). A more detailed description of these lines is provided in a forthcoming paper (Holtzman et al., in prep.). In particular, a mix of heavily $\mathrm{CN}$-cycle and $\alpha$-poor MARCS models were used; the same molecular lines as adopted by Smith et al. (2013) were also employed to determine the $\mathrm{C}, \mathrm{N}$, and $\mathrm{O}$ abundances. In addition, we adopted the $\mathrm{C}, \mathrm{N}$, and $\mathrm{O}$ abundances that satisfy the fitting of all molecular lines consistently; i.e., we first derive ${ }^{16} \mathrm{O}$ abundances from ${ }^{16} \mathrm{OH}$ lines, then derive ${ }^{12} \mathrm{C}$ from ${ }^{12} \mathrm{C}^{16} \mathrm{O}$ lines and ${ }^{14} \mathrm{~N}$ from ${ }^{12} \mathrm{C}^{14} \mathrm{~N}$, lines, and the $\mathrm{CNO}$ abundances are derived several times to minimize the $\mathrm{OH}, \mathrm{CO}$, and $\mathrm{CN}$ dependences (see, e.g., Smith et al. 2013; Fernández-Trincado et al. 2019a).

We additionally evaluated the possibility of applying the approach of fixing $T_{\mathrm{eff}}^{\mathrm{pho}}$ and $\log g$ to values determined independently of spectroscopy to check for any significant deviation in the chemical abundances. For this, the photometric effective temperature, $T_{\text {eff }}^{\text {pho }}=4806.6 \mathrm{~K}$, was calculated from the $J_{2 \text { MASS }}$ to $K_{s, 2 \text { MASS }}$ color relation using the methodology presented in González Hernández \& Bonifacio (2009); for 2M12451043+1217401 we adopt $J_{2 \text { MASS }}-K_{s, 2 \text { MASS }}=0.637 \mathrm{mag}$ and $[\mathrm{Fe} / \mathrm{H}]=-1.09$. The photometry is extinction-corrected using the Rayleigh Jeans color excess (RJCE) method (see Majewski et al. 2011), which leads to $\left\langle A_{K}^{\mathrm{WISE}}\right\rangle \sim 0.023 \mathrm{mag}$. The resulting temperature, $T_{\mathrm{eff}}^{\mathrm{pho}}=$ $4806.6 \mathrm{~K}$, is in very good agreement with the spectroscopic temperatures from ASPCAP and Payne-APOGEE. In conclusion, this small $T_{\text {eff }}$ discrepancy does not affect our results. In the following, our analysis is restricted to the atmospheric parameters as listed in Table 1. The same table also lists the abundance measurements in this star obtained with the BACCHUS code and compared to ASPCAP and Payne-APOGEE pipelines. We also list the total error bar on our measurements, which is based on contributions from the statistical and systematic uncertainties.
Table A.1. Atomic lines and derived log abundances for the light elements $\mathrm{Fe}, \mathrm{Al}, \mathrm{Mg}$, and $\mathrm{Si}$, and the heavy element $\mathrm{Ce}$.

\begin{tabular}{|c|c|c|}
\hline Element & $\lambda^{\mathrm{air}}(\AA)$ & $\log (\epsilon)$ \\
\hline \multirow[t]{39}{*}{$\mathrm{Fe} I$} & 15207.5 & 6.194 \\
\hline & 15245.0 & 6.317 \\
\hline & 15294.6 & 6.225 \\
\hline & 15394.7 & 6.377 \\
\hline & 15500.8 & 6.210 \\
\hline & 15501.3 & 6.216 \\
\hline & 15531.8 & 6.443 \\
\hline & 15534.2 & 6.415 \\
\hline & 15588.3 & 6.515 \\
\hline & 15591.5 & 6.308 \\
\hline & 15604.2 & 6.180 \\
\hline & 15621.7 & 6.324 \\
\hline & 15632.0 & 6.296 \\
\hline & 15662.0 & 6.332 \\
\hline & 15723.6 & 6.356 \\
\hline & 15769.1 & 6.583 \\
\hline & 15769.4 & 6.583 \\
\hline & 15774.1 & 6.400 \\
\hline & 15895.2 & 6.456 \\
\hline & 15904.3 & 6.419 \\
\hline & 15920.6 & 6.324 \\
\hline & 15964.9 & 6.460 \\
\hline & 15967.7 & 6.414 \\
\hline & 15980.7 & 6.331 \\
\hline & 16006.8 & 6.356 \\
\hline & 16007.1 & 6.357 \\
\hline & 16040.7 & 6.437 \\
\hline & 16042.7 & 6.172 \\
\hline & 16071.4 & 6.453 \\
\hline & 16125.9 & 6.505 \\
\hline & 16153.2 & 6.419 \\
\hline & 16179.6 & 6.379 \\
\hline & 16195.1 & 5.969 \\
\hline & 16284.8 & 6.489 \\
\hline & 16517.2 & 6.402 \\
\hline & 16524.5 & 6.427 \\
\hline & 16561.8 & 6.483 \\
\hline & 16645.9 & 6.300 \\
\hline & 16665.5 & 6.468 \\
\hline$\langle\mathrm{A}(\mathrm{Fe})\rangle \pm \sigma_{\text {mean }}$ & & $6.37 \pm 0.12$ \\
\hline \multirow[t]{3}{*}{$\mathrm{Al} \mathrm{I}$} & 16719.0 & 5.253 \\
\hline & 16750.6 & 5.237 \\
\hline & 16763.4 & 5.503 \\
\hline$\langle\mathrm{A}(\mathrm{Al})\rangle \pm \sigma_{\text {mean }}$ & & $5.33 \pm 0.12$ \\
\hline \multirow[t]{3}{*}{$\mathrm{Mg} \mathrm{I}$} & 15740.7 & 6.586 \\
\hline & 15748.9 & 6.556 \\
\hline & 15765.8 & 6.537 \\
\hline$\langle\mathrm{A}(\mathrm{Mg})\rangle \pm \sigma_{\text {mean }}$ & & $6.56 \pm 0.02$ \\
\hline \multirow[t]{10}{*}{ Si I } & 15376.8 & 6.450 \\
\hline & 15557.8 & 6.629 \\
\hline & 15884.5 & 6.475 \\
\hline & 15960.1 & 6.623 \\
\hline & 16060.0 & 6.738 \\
\hline & 16094.8 & 6.728 \\
\hline & 16215.7 & 6.762 \\
\hline & 16241.8 & 6.775 \\
\hline & 16680.8 & 6.570 \\
\hline & 16828.2 & 6.766 \\
\hline$\langle\mathrm{A}(\mathrm{Si})\rangle \pm \sigma_{\text {mean }}$ & & $6.65 \pm 0.12$ \\
\hline \multirow[t]{3}{*}{ Ce II } & 15784.8 & 1.029 \\
\hline & 15958.4 & 1.055 \\
\hline & 16376.5 & 0.911 \\
\hline$\langle\mathrm{A}(\mathrm{Ce})\rangle \pm \sigma_{\text {mean }}$ & & $0.998 \pm 0.06$ \\
\hline
\end{tabular}


Table A.2. Molecular features and log abundances used to derive $\mathrm{C}, \mathrm{N}$, and $\mathrm{O}$.

\begin{tabular}{|c|c|c|}
\hline Element & $\lambda^{\mathrm{air}}(\AA)$ & $\log (\epsilon)$ \\
\hline \multirow[t]{2}{*}{${ }^{12} \mathrm{C}$ from ${ }^{12} \mathrm{C}^{16} \mathrm{O}$ lines } & 15578.0 & 7.532 \\
\hline & 16185.5 & 7.202 \\
\hline \multicolumn{2}{|l|}{$\langle\mathrm{A}(\mathrm{C})\rangle \pm \sigma_{\text {mean }}$} & $7.37 \pm 0.17$ \\
\hline \multirow[t]{19}{*}{${ }^{14} \mathrm{~N}$ from ${ }^{12} \mathrm{C}^{14} \mathrm{~N}$ lines } & 15158.0 & 6.980 \\
\hline & 15165.4 & 7.483 \\
\hline & 15185.6 & 7.152 \\
\hline & 15210.2 & 7.228 \\
\hline & 15222.0 & 7.433 \\
\hline & 15251.8 & 7.118 \\
\hline & 15284.5 & 7.494 \\
\hline & 15309.0 & 7.442 \\
\hline & 15317.6 & 7.591 \\
\hline & 15328.4 & 7.226 \\
\hline & 15363.5 & 7.483 \\
\hline & 15447.0 & 7.432 \\
\hline & 15462.4 & 7.400 \\
\hline & 15466.2 & 7.587 \\
\hline & 15495.0 & 7.489 \\
\hline & 15514.0 & 7.420 \\
\hline & 15581.0 & 7.264 \\
\hline & 15659.0 & 7.592 \\
\hline & 16244.0 & 7.507 \\
\hline \multicolumn{2}{|l|}{$\langle\mathrm{A}(\mathrm{N})\rangle \pm \sigma_{\text {mean }}$} & $7.39 \pm 0.17$ \\
\hline \multirow[t]{11}{*}{${ }^{16} \mathrm{O}$ from ${ }^{16} \mathrm{OH}$ lines } & 15409.2 & 8.153 \\
\hline & 15568.8 & 8.003 \\
\hline & 16052.8 & 7.748 \\
\hline & 16055.5 & 7.660 \\
\hline & 16255.0 & 7.935 \\
\hline & 16312.6 & 8.287 \\
\hline & 16534.6 & 7.893 \\
\hline & 16650.0 & 8.149 \\
\hline & 16656.0 & 8.160 \\
\hline & 16704.5 & 8.215 \\
\hline & 16898.9 & 8.153 \\
\hline$\langle\mathrm{A}(\mathrm{O})\rangle \pm \sigma_{\text {mean }}$ & & $8.03 \pm 0.19$ \\
\hline
\end{tabular}

Table A.3. Abundance determination sensitivity to the stellar parameters from our present measurements.

\begin{tabular}{lccc}
\hline \hline$\Delta[\mathrm{X} / \mathrm{H}]$ & $T_{\text {eff }} \pm 100 \mathrm{~K}$ & $\log g \pm 0.3$ & $\xi_{t} \pm 0.05 \mathrm{~km} \mathrm{~s}^{-1}$ \\
\hline $\mathrm{Fe}$ & 0.03 & 0.05 & 0.002 \\
$\mathrm{C}$ & 0.14 & 0.06 & 0.002 \\
$\mathrm{~N}$ & 0.11 & 0.09 & 0.001 \\
$\mathrm{O}$ & 0.09 & 0.03 & 0.007 \\
$\mathrm{Al}$ & 0.04 & 0.04 & 0.004 \\
$\mathrm{Mg}$ & 0.08 & 0.09 & 0.060 \\
$\mathrm{Si}$ & 0.02 & 0.06 & 0.004 \\
$\mathrm{Ce}$ & 0.05 & 0.19 & 0.002 \\
\hline
\end{tabular}

\section{Appendix B: Variability}

We use multi-epoch radial velocity measurements available in the APOGEE-2 DR14 database. 2M12451043+1217401 was observed multiple times in a series of 25 "visits" to meet the $\mathrm{S} / \mathrm{N}$ requirements of the APOGEE-2 survey. The radial veloc-
Table B.1. APOGEE observations.

\begin{tabular}{lccc}
\hline \hline Julian date & $\mathrm{RV}^{\dagger}\left(\mathrm{km} \mathrm{s}^{-1}\right)$ & $\sigma\left(\mathrm{km} \mathrm{s}^{-1}\right)$ & $S / N\left(\mathrm{pixel}^{-1}\right)$ \\
\hline 2457059.94391 & -88.85 & 0.16 & 20 \\
2457060.91321 & -89.43 & 0.15 & 20 \\
2457062.99631 & -89.45 & 0.15 & 20 \\
2457064.89865 & -90.54 & 0.17 & 18 \\
2457114.79574 & -92.80 & 0.22 & 17 \\
2457118.85652 & -92.28 & 0.43 & 8 \\
2457121.82661 & -92.55 & 0.19 & 16 \\
2457122.80708 & -93.23 & 0.14 & 23 \\
2457141.73865 & -93.40 & 0.21 & 15 \\
2457142.73487 & -93.09 & 0.17 & 18 \\
2457148.68704 & -94.02 & 0.19 & 16 \\
2457167.65509 & -92.67 & 0.79 & 5 \\
2457449.88122 & -74.01 & 0.12 & 26 \\
2457465.78237 & -74.55 & 0.12 & 22 \\
2457468.79933 & -73.70 & 0.14 & 22 \\
2457472.79234 & -74.23 & 0.14 & 23 \\
2457473.88693 & -73.25 & 0.16 & 20 \\
2457475.83189 & -73.32 & 0.13 & 22 \\
2457492.72504 & -73.91 & 0.12 & 23 \\
2457496.80519 & -73.52 & 0.21 & 14 \\
2457499.79405 & -73.47 & 0.16 & 19 \\
\hline $2457504.78284 \ddagger$ & -69.84 & 0.89 & 4 \\
\hline 2457530.70246 & -73.99 & 0.19 & 16 \\
2457533.71368 & -73.81 & 0.11 & 25 \\
2457534.73149 & -74.29 & 0.19 & 14 \\
\hline
\end{tabular}

Notes. ${ }^{(\dagger)}$ Reduced heliocentric JD. ${ }^{(\ddagger)}$ Values omitted for spectra with $S / N<5$.

ities for each visit are determined using an iterative scheme, i.e., the individual visit spectra are combined using initial guesses for the relative radial velocities into a co-added spectrum, which is then used to re-derive the relative visit velocities (see, e.g., Nidever et al. 2015; Price-Whelan et al. 2018).

Generally, observations from the APOGEE-2 survey have a relatively short ( $\lesssim 6$ months) baseline, which is a potential limitation to enabling the detection of N-rich stars formed through the binary channel. However, we find that the large scatter $\left(>9.92 \mathrm{~km} \mathrm{~s}^{-1}\right)$ shown by the measured radial velocities in $2 \mathrm{M} 12451043+1217401$ implies the clear existence of at least one companion. So far, no evidence of strong radial velocity variations has been found in stars with nitrogen over-abundances in the APOGEE survey (see, e.g., Martell et al. 2016; FernándezTrincado et al. 2016b, 2017; Schiavon et al. 2017). Therefore, establishing the presence of radial velocity variation among these stars would be required to understand if many, or all such objects, formed through the binary channel.

Although APOGEE-2 observed our star during multiple "visits", we omitted one observation from our radial velocity analysis with a $\mathrm{S} / \mathrm{N}$ below 4 per pixel. The observations are summarized in Table B.1, which lists the APOGEE radial velocities, their uncertainties, and the $\mathrm{S} / \mathrm{N}$ per pixel for each epoch.

We conducted a search for the orbital period using the genetic algorithm PIKAIA (Charbonneau 1995) to determine the orbital parameters that best fit the available data. Following the analysis described by Mennickent et al. (2012, 2018a,b) we minimize $\chi^{2}$ defined as

$\chi^{2}(P, \tau, \omega, e, K, \gamma)=\frac{1}{N-6} \sum_{j=1}^{n}\left(\frac{V_{J}-V\left(t_{j}, P, \tau, \omega, e, K, \gamma\right)}{\sigma_{j}}\right)^{2}$, 


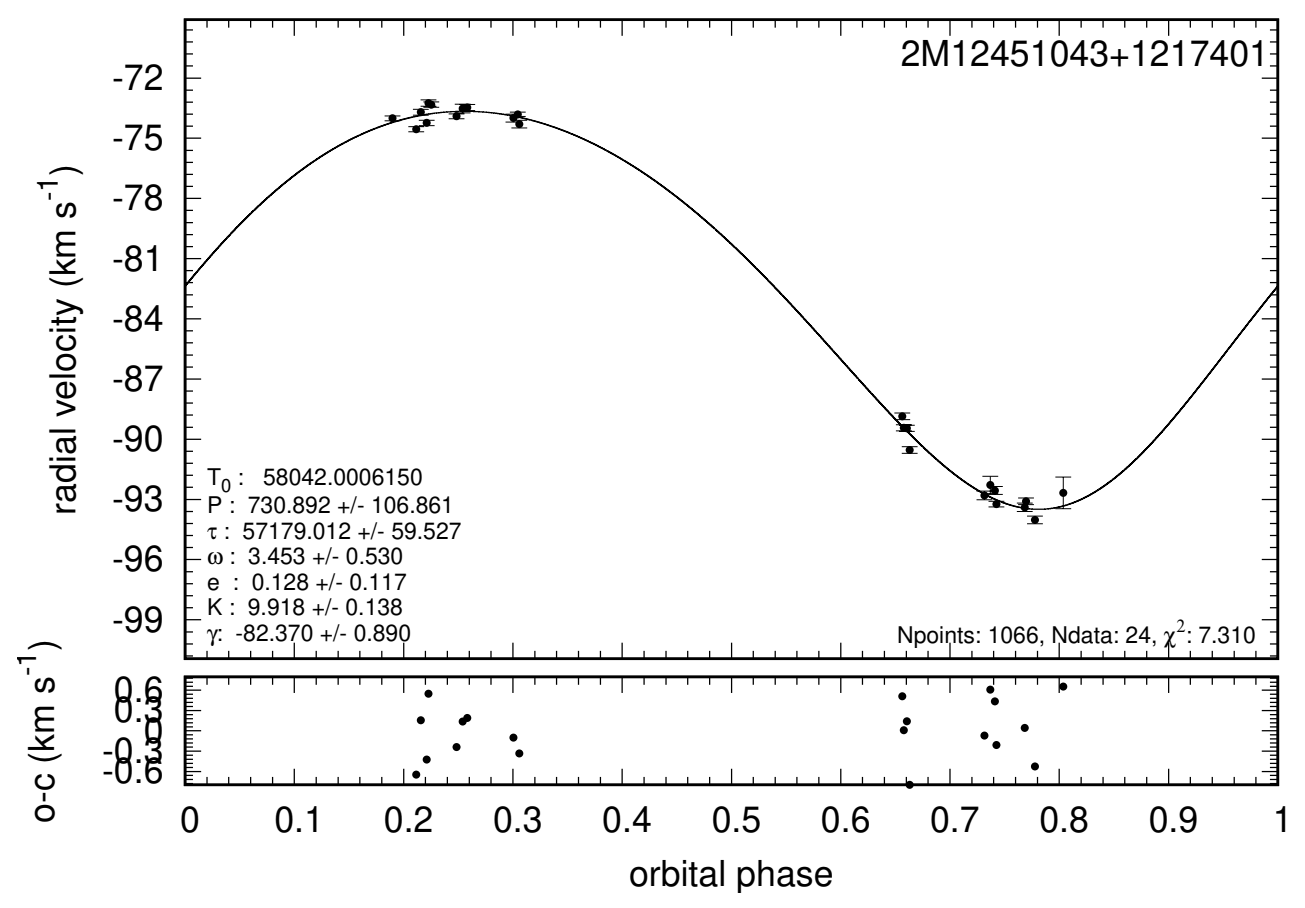

Fig. B.1. Radial velocity measurements for $2 \mathrm{M} 12451043+1217401$ from 24 visits of the APOGEE-2 survey (top panel) with the best fit, with the residual velocities (bottom panel).
Table B.2. Orbital elements for the donor of 2M12451043+1217401 obtained by minimization of the $\chi^{2}$ parameter given by Eq. (B.1).

\begin{tabular}{lccc}
\hline \hline Parameter & Best value & Lower limit & Upper limit \\
\hline$P_{o}(\mathrm{~d})$ & $730.89 \pm 106.86$ & 679.61 & 893.33 \\
$\tau^{*}$ & $57179.0117 \pm 59.5272$ & 57129.9301 & 57248.9845 \\
$\omega$ & $3.45 \pm 0.53[\mathrm{rad}]$ & 2.961 & 4.022 \\
$e$ & $0.1276 \pm 0.1174$ & 0.0569 & 0.2916 \\
$K_{2}\left(\mathrm{~km} \mathrm{~s}^{-1}\right)$ & $9.92 \pm 0.14$ & 9.856 & 10.131 \\
$\gamma\left(\mathrm{km} \mathrm{s}^{-1}\right)$ & $-82.37 \pm 0.89$ & -83.22 & -81.44 \\
\hline
\end{tabular}

Notes. The value $\tau^{*}=\tau-2450000$ and the limits of the confidence intervals within one standard deviation $(1 \sigma)$ are given.

where $N$ is the number of observations, and $V_{j}$ and $V$ are the observed and calculated velocities in time $t_{j}$. The fit velocity is written as

$V(t)=\gamma+K((\omega+\theta(t))+e \cos (\omega))$

where $\theta$ is the true anomaly obtained by solving the equations:

$\tan \left(\frac{\theta}{2}\right)=\sqrt{\frac{1+e}{1-e}} \tan \left(\frac{E}{2}\right)$

$E-e \sin (E)=\frac{2 \pi}{P}(t-\tau)$,

where $E$ is the eccentric anomaly. We constrained the eccentricity between 0 and $1, \omega$ between 0 and $2 \pi, \tau$ between the minimum HJD and this value plus the period, $K$ between 0 and $\left(V_{\max }-V_{\min }\right)$, and $\gamma$ between $V_{\min }$ and $V_{\max }$.

In order to estimate the errors for the results obtained from PIKAIA, we proceeded to calculate the confidence intervals for the region corresponding to $68.26 \%$ of the sample $(1 \sigma)$. The results are listed in Table B.2. The fit produces a good match to the available data (residuals $\approx 1 \mathrm{~km} \mathrm{~s}^{-1}$; see bottom panel in Fig. B.1).
It is important to note that $2 \mathrm{M} 12451043+1217401$ was identified as a "bimodal binary" in the bimodal period samplings of The Joker code in Price-Whelan et al. (2018), correponding to periods between $P \simeq 650-950$ days and eccentricity between $\simeq 0.07-0.3:\left(P_{1}, e_{1}\right)=(939.80,0.31)$ days and $\left(P_{2}, e_{2}\right)=(689.85$ days, 0.07$)$. The best-fit orbital parameters from PIKAIA are an orbital period of $730.89 \pm 106.86$ days, a velocity semi-amplitude of $9.92 \pm 0.14 \mathrm{~km} \mathrm{~s}^{-1}$, and an eccentricity of $0.1276 \pm 0.1174$. These are in acceptable agreement with the shorter period reported by Price-Whelan et al. (2018).

Visual inspection of the spectrum of 2M12451043+1217401 does not reveal any obvious signs of binary interaction, such as emission lines from an accretion disk. If this is a post-masstransfer binary system, the primary would have evolved into a white dwarf by this point, making it very difficult to detect in the $\mathrm{H}$ band. Alternately, if there was very strong mass loss from the primary, the binary system could have disrupted or significantly widened. For the case of SB1 binaries, where only one component is detected in the spectrum, the mass information is contained in a single function, called the mass function, defined as

$f=\frac{\left(m_{1}^{3}\right)\left(\sin ^{3}(i)\right)}{\left(m_{1}+m_{2}\right)^{2}}=1.0361 \times 10^{-7}\left(1-e^{2}\right)^{3 / 2}\left(\frac{K_{2}}{\mathrm{~km} \mathrm{~s}^{-1}}\right)^{3} \frac{P_{o}}{\text { days }} M_{\odot}$.

This corresponds to the minimum mass of the unseen companion, labeled with subscript 1 , in this case $0.07 M_{\odot}$. Since the system inclination is unknown, several solutions are possible for the mass of the unseen companion. Assuming $1 M_{\odot}$ for the detected star (Price-Whelan et al. 2018), we summarize our restrictions for the mass of the unseen companion in Fig. B.2.

From the system mass function (Eq. (B.5)), and assuming a $1 M_{\odot}$ for the detected component, we can determine the mass of the unseen companion from the intersection of two functions of the form $m_{1}^{3} \sin ^{3} i$ and $g\left(P, K_{2}, e\right) \times\left(m_{1}+m_{2}\right)^{2}$, where $g$ is a function of the orbital period, radial velocity half-amplitude, and orbital eccentricity. These functions are plotted for our system 


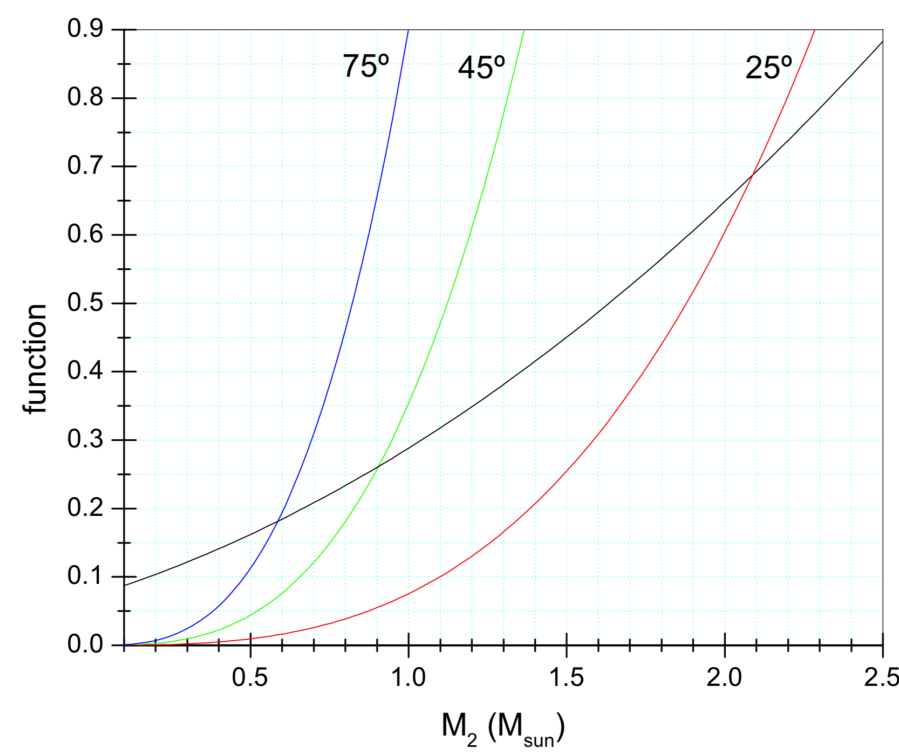

Fig. B.2. Intersection between the black and reference lines give the estimated mass for the unseen companion at different inclination angles.

for three different angles showing a possible range of masses for the companion (see Fig. B.2).

\section{Appendix C: Dynamical behavior}

We present a first attempt to predict the probable orbit of the newly discovered N-rich binary across the Milky Way. To do this, we used a current orbital integration model in an, as much as possible, realistic gravitational potential, which fits the structural and dynamical parameters of the Galaxy based on the recent knowledge of our Milky Way. For the computations in this work, we employed the rotating "boxy/peanut" bar model of the novel galactic potential model, called GravPot $16^{2}$, along with other composite stellar components. The considered structural parameters of our bar model, for example, mass $\sim 1.1 \times 10^{10} M_{\odot}$ ), present-day orientation $\sim 20^{\circ}$ ), and pattern speeds $\Omega$ between $35 \mathrm{~km} \mathrm{~s}^{-1} \mathrm{kpc}^{-1}$ and $50 \mathrm{~km} \mathrm{~s}^{-1} \mathrm{kpc}^{-1}$ in steps of $5 \mathrm{~km} \mathrm{~s}^{-1} \mathrm{kpc}^{-1}$ ), which is within observational estimations.

For reference, the galactic convention adopted by this work is: $X$-axis is oriented toward $l=0^{\circ}$ and $b=0^{\circ}$, the $Y$-axis is oriented toward $l=90^{\circ}$ and $b=0^{\circ}$, and the disk rotates toward $l=90^{\circ}$; the velocity components are also oriented along these directions. In this convention, the Sun's orbital velocity vector
Table C.1. Phase-space data.

\begin{tabular}{ll}
\hline \hline Coordinates & $(J 2000)$ \\
$(\alpha, \delta)$ & $\left(191^{\circ} .293486,12^{\circ} .294486\right)$ \\
$(l, b)$ & $\left(296^{\circ} .973729363,75^{\circ} .0936328462\right)$ \\
Heliocentric distance & {$[\mathrm{kpc}]$} \\
& $(4.54 \pm 0.93)^{(a)}$ \\
& $(13.02 \pm 1.49)^{(b)}$ \\
$\langle\mathrm{RV}\rangle \pm \sigma_{\mathrm{RV}}$ & {$\left[\mathrm{km} \mathrm{s}^{-1}\right]$} \\
& $(-82.8 \pm 9.9)^{(c)}$ \\
Proper motions & $\left(\mu_{\alpha} \cos \delta, \mu_{\delta}\right)$ \\
& {$\left[\mathrm{mas} \mathrm{yr}^{-1}\right]$} \\
& $(-1.32 \pm 0.12,-4.19 \pm 0.06)^{(d)}$ \\
&
\end{tabular}

Notes. ${ }^{(a)}$ Estimated distance computed by Bailer-Jones et al. (2018); ${ }^{(b)}$ estimated distance computed using the StarHorse code (Queiroz et al. 2018); ${ }^{(c)}$ the average radial velocity of the binary system computed from the 24 visits of the APOGEE/DR14 spectra (see text); ${ }^{(d)}$ absolute proper motions from Gaia Collaboration (2018).

are $\left[U_{\odot}, V_{\odot}, W_{\odot}\right]=[11.1,12.24,7.25] \mathrm{km} \mathrm{s}^{-1}$ (Brunthaler et al. 2011). The model has been rescaled to the Sun's galactocentric distance, $8.3 \mathrm{kpc}$, and a local rotation velocity of $239 \mathrm{~km} \mathrm{~s}^{-1}$. For computation of the galactic orbits, we have adopted a simple Monte Carlo approach and the Runge-Kutta algorithm of seventh-eight order elaborated by Fehlberg (1968). The uncertainties in the input data (e.g., distances, proper motions, and line-of-sight velocity errors listed in Table C.1) are assumed to follow a Gaussian distribution and were propagated as $1 \sigma$ variations in a Gaussian Monte Carlo resampling. We sampled a half million orbits, computed backward in time for 3 Gyr. Figure C. 1 shows the probability densities of the resulting orbits projected on the equatorial (left column) and meridional (right column) galactic planes, in the non-inertial reference frame where the bar is at rest. The orbital path (adopting central values) is shown by the black line in the same figure. The green and yellow colors correspond to more probable regions of the space, which are crossed more frequently by the simulated orbits.

We derive the distance to $2 \mathrm{M} 12451043+1217401$ using StarHorse ${ }^{3}$, which is a Bayesian distance estimator initially developed for APOGEE stars (Queiroz et al. 2018). For completeness, we provide dynamical solutions and orbit calculations for both the StarHorse distance of $13 \mathrm{kpc}$ and the Bailer-Jones et al. (2018) distance of $4.5 \mathrm{kpc}$. The two columns on the left of Fig. C. 1 shows possible orbits given the StarHorse distance and different bar speeds $\left(35,40,45\right.$, and $\left.50 \mathrm{~km} \mathrm{~s}^{-1} \mathrm{kpc}\right)$, and the two columns on the right show the same figures for the Bailer-Jones et al. (2018) distance. 

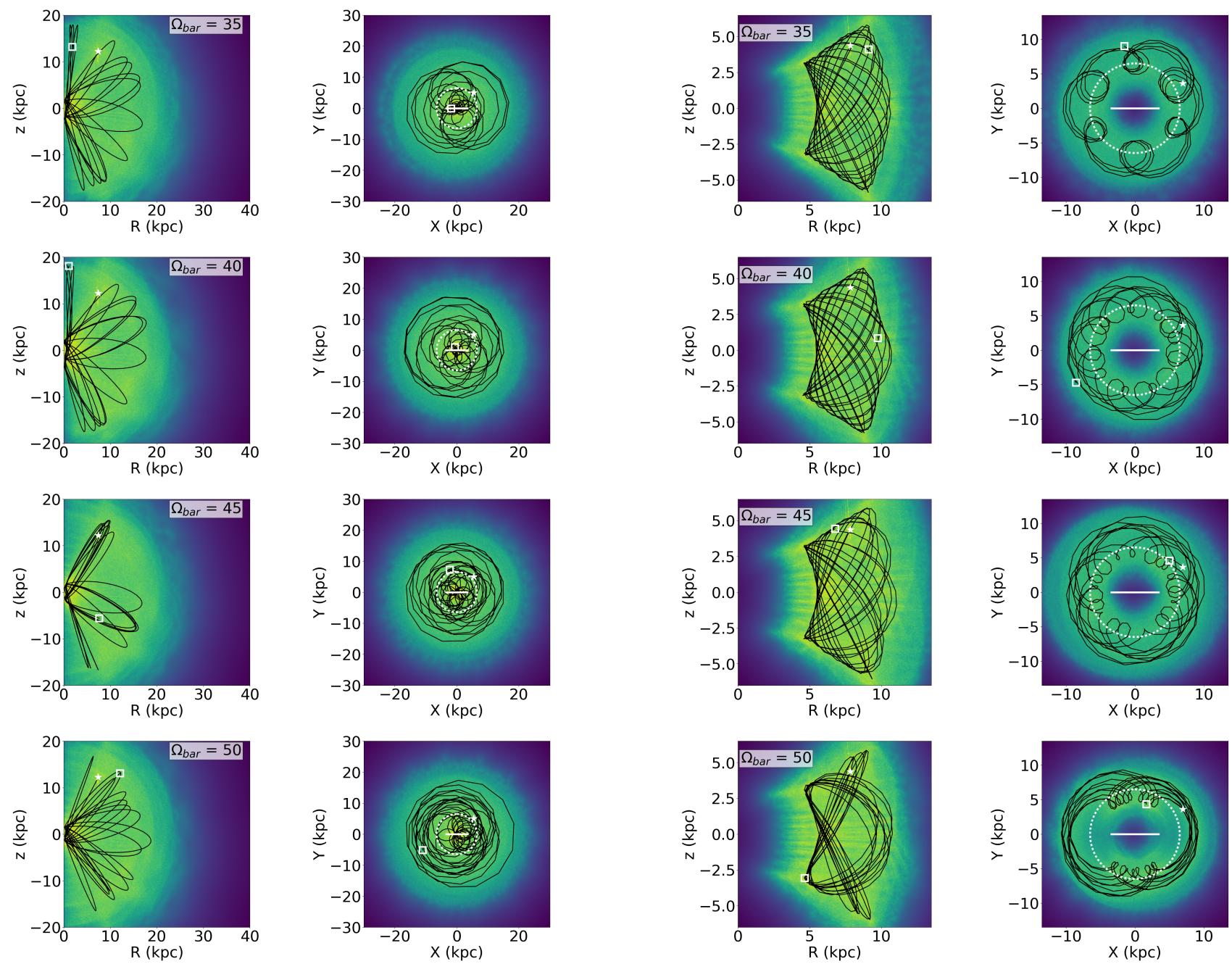

Fig. C.1. Kernel density estimate smoothed distribution of simulated orbits, showing the probability densities of the resulting orbits projected on the equatorial and meridional galactic planes in the non-inertial reference frame where the bar is at rest. The green and yellow colors correspond to more probable regions of the space, which are crossed more frequently by the simulated orbits. The black line is the orbit of $2 \mathrm{M} 12451043+1217401$ adopting the central inputs. The small white star indicates the present position of the N-rich star, whereas the white square indicates its final position. In all orbit panels, the white dotted circle shows the location of the co-rotation radius (CR), the horizontal white solid line shows the extension of the bar. Columns 1 and 2 show the results adopting the estimated distance from the StarHorse code, while columns 3 and 4 for estimated distance from Bailer-Jones et al. (2018). 